\section{Schnelle Hilfe in der Akuttherapie durch Olanzapin i.m.}

$\mathrm{N}$ eben einer starken Agitation zeigen sich akut psychotische Patienten häufig wenig krankheitseinsichtig. Hinzu kommt nicht selten das Risiko der Eigen- und Fremdgefährdung. Hier ist rasches Handeln gefragt. Bislang wird in der Akuttherapie der Schizophrenie und Manie mangels Alternativen meist auf hochpotente klassische Neuroleptika zurückgegriffen, wobei man das Risiko für extrapyramidal-motorische Nebenwirkungen und somit eine langfristige Gefährdung der Compliance in Kauf nimmt. Ein Beispiel aus Hessen zeigt, dass durch die neue parenterale Darreichungsform von Olanzapin jetzt auch Patienten, die eine orale Medikation verweigern, von der guten Wirksamkeit und Verträglichkeit des atypischen Neuroleptikums profitieren können.

\section{Aufnahme}

Die Aufnahme eines 42-jährigen, 92 kg schweren, männlichen Patienten aus dem betreuten Wohnen erfolgte wegen unkooperativen Verhaltens sowie Feindseligkeit mit verbaler Aggression gegen Mitbewohner und Betreuer. Der Patient kam freiwillig und wurde wegen ausgeprägter psychomotorischer Unruhe, innerer Gespanntheit und Feindseligkeit in die geschlossene Abteilung aufgenommen.

\section{Anamnese und Biographie}

Seit 20 Jahren liegt bei dem Patienten eine bekannte paranoidhalluzinatorische Schizophrenie mit insgesamt zwölf stationären Aufenthalten vor. Seine Schulausbildung hat er nach der mittleren Reife abgebrochen und versuchte eine Lehre als Installateur sowie später als Kfz-Mechaniker. Beide wurden nicht abgeschlossen. Bis zu seinem 28. Lebensjahr wohnte der Patient bei den Eltern; danach in Wohnheimen für psychisch Kranke. Der Patient ist in keiner aktuellen Partnerschaft, hatte keine längeren Beziehungen und ist weder verheiratet noch hat er Kinder.
Die letzten vier Wochen vor Beginn der stationären Behandlung litt der Patient unter zunehmender innerer Unruhe, zerfahrenem Denken, Fremdbeeinflussungserleben, Ich-Erlebnisstörungen, paranoiden Gedanken, imperativen Stimmen und Feindseligkeit. Dabei kam es zu zunehmendem Cannabis- und Alkoholmissbrauch. Hierunter kam es zu verbalen Entgleisungen mit tätlichen Angriffen gegen Mitbewohner des betreuten Wohnens und gegen Mitarbeiter der betreuenden Tagesklinik.

Letzte Medikation: FluphenazinDepot $50 \mathrm{mg}$ alle 3 Wochen, Quetiapin 2 x $300 \mathrm{mg} /$ die.

\section{Psychopathologischer \\ Aufnahmebefund}

Der Patient ist wach und ansprechbar und in allen Qualitäten ausreichend orientiert. Seine Stimmung ist aggressiv, gereizt und impulsiv. Der Antrieb ist rastlos gesteigert, getrieben und psychomotorisch erregt. Der Patient fühlt sich verfolgt, vom Personal des Krankenhauses abgehört und überwacht sowie durch einen Pfleger fremdbeeinflusst. Er hört imperative Stimmen, die ihm sagen, er solle keine Tabletten mehr einnehmen, da diese vergiftet seien. Außerdem ist er leicht reizbar und verbal fremdaggressiv gegen Mitpatienten, von denen er sich bedroht fühlt.

\section{Therapie und Verlauf}

Als die Situation derart eskaliert, dass der Patient droht, einen Krankenpfleger „mit dem Messer abzustechen“, wird der Patient überzeugt, dass Hilfe durch ein Medikament unumgänglich ist. Da er jegliche orale Medikation kategorisch ablehnt, akzeptiert er schließlich eine i.m.-Applikation von Olanzapin (10 mg Zyprexa ${ }^{\circledR}$ i.m.), nachdem ihm zugesichert wurde, dass es hierbei zu keinen extrapyramidal-motorischen Nebenwirkungen (z.B. Frühdyskinesien) kommen kann.

Zum Zeitpunkt der Injektion wurde die PANSS-Subscore aus den Items Gespanntheit (7 Punkte), feh- lende Kooperation (5 Punkte), Feindseligkeit (7 Punkte), mangelnde Impulskontrolle (5 Punkte) und psychomotorische Erregung (7 Punkte) mit insgesamt 31 Punkten bewertet. Bereits 30 Minuten nach der Injektion zeigte sich der Patient in allen Items deutlich gebessert (Gesamtscore 20 Punkte).

Seitens des Pflegepersonals wird eine deutliche Besserung der psychomotorischen Erregung, deutliche Entspannung, nachgelassene Feindseligkeit und verbesserte Führbarkeit berichtet. Nach 30 Minuten ist der Patient von seinem paranoiden Erleben soweit distanziert, dass eine orale Medikation mit Olanzapin $3 \mathrm{x}$ $10 \mathrm{mg}$ VeloTab $^{\mathrm{TM}}{ }^{*}$ akzeptiert wird.

Am nächsten Morgen - nach komplikationsloser Nacht - stellte sich die Situation trotz der oralen Medikation wieder verschärft dar, ohne jedoch das Niveau des Vortages zu erreichen. Der Patient bittet während der täglichen Visite um eine zweite Olanzapin i.m.-Injektion, da die vom Vortage so „gut getan“ habe.

Er bekommt trotz der oralen Gabe von 30 mg Olanzapin* eine weitere i.m.-Spritze (10 mg). Innerhalb von 30 Minuten ist der Patient wiederum deutlich entspannter, geradezu freundlich und ausgeglichen, distanziert von wahnhaftem Erleben, kooperativ und ohne jegliche Feindseligkeit oder Aggressivität. Drei Tage nach der Aufnahme wurde die Medikation auf eine Tagesdosis von $20 \mathrm{mg}$ Olanzapin reduziert. Unter dieser Dosierung konnte der Patient nach 18 Tagen vollständig remittiert entlassen werden.

\section{Fazit}

Die intramuskuläre Darreichungsform von Olanzapin stellt sich als ein schnell wirksames, zuverlässiges und gut verträgliches Präparat zur Behandlung psychotisch motivierter Akutsituationen dar, das beim Patienten besonders wegen der guten Verträglichkeit hohe Akzeptanz erfährt.

\section{Korrespondenzadresse:}

Prof. Dr. med. Dr. rer. nat. Joachim Röschke St. Valentinushaus

Klinik für Psychiatrie und Psychotherapie Kiedrich

\footnotetext{
* In dieser Dosierung nicht zugelassen. Zugelassener Dosierungsbereich 2,5-20 mg/die
} 